\title{
How stellar activity affects the size estimates of extrasolar planets
}

\author{
S. Czesla, K. F. Huber, U. Wolter, S. Schröter, and J. H. M. M. Schmitt
}

\author{
Hamburger Sternwarte, Universität Hamburg, Gojenbergsweg 112, 21029 Hamburg, Germany \\ e-mail: stefan.czesla@hs.uni-hamburg.de
}

Received 8 May 2009 / Accepted 1 July 2009

\section{ABSTRACT}

\begin{abstract}
Light curves have long been used to study stellar activity and have more recently become a major tool in the field of exoplanet research. We discuss the various ways in which stellar activity can influence transit light curves, and study the effects using the outstanding photometric data of the CoRoT-2 exoplanet system. We report a relation between the "global" light curve and the transit profiles, which turn out to be shallower during high spot coverage on the stellar surface. Furthermore, our analysis reveals a color dependence of the transit light curve compatible with a wavelength-dependent limb darkening law as observed on the Sun. Taking into account activity-related effects, we redetermine the orbit inclination and planetary radius and find the planet to be $\approx 3 \%$ larger than reported previously. Our findings also show that exoplanet research cannot generally ignore the effects of stellar activity.
\end{abstract}

Key words. techniques: photometric - stars: activity - starspots - stars: individual: CoRoT-2a - planetary systems

\section{Introduction}

The brightness distribution on the surface of active stars is both spatially inhomogeneous and temporally variable. The state and evolution of the stellar surface structures can be traced by the rotational and secular modulation of the observed photometric light curve. In the field of planet research, light curves including planetary transits are of particular interest, since they hold a wealth of information about both the planet and its host star.

The outstanding quality of the space-based photometry provided by the CoRoT mission (e.g., Auvergne et al. 2009) provides stellar light curves of unprecedented precision, temporal cadence and coverage. While primarily designed as a planet finder, the CoRoT data are also extremely interesting in the context of stellar activity. Lanza et al. (2009) demonstrated the information content to be extracted from these light curves in the specific case of CoRoT-2a. This star is solar-like in mass and radius, but rotates faster at a speed of $v \sin (i)=11.85 \pm 0.50 \mathrm{~km} \mathrm{~s}^{-1}$ (Bouchy et al. 2008). Its rotation period of $\approx 4.52$ days was deduced from slowly evolving active regions, which dominate the photometric variations. Thus, CoRoT-2a is a very active star by all standards. Even more remarkably, CoRoT-2a is orbited by a giant planet (Alonso et al. 2008), which basically acts as a shutter scanning the surface of CoRoT-2a along a well defined latitudinal band.

The transiting planetary companion provides a key to understanding the surface structure of its host star. While previous analyses have either ignored the transits (Lanza et al. 2009) or the "global" light curve (Wolter et al. 2009), we show that there is a relation between the transit shape and the global light curve, which cannot generally be neglected in extrasolar planet research.

\section{Observations and data reduction}

Alonso et al. (2008) discovered the planet CoRoT-2b using the photometric CoRoT data (see Table 1). Its host star has a spectral type of G7V with an optical (stellar) companion too close
Table 1. Stellar/planetary parameters of CoRoT-2a/b.

\begin{tabular}{lcc}
\hline \hline Star $^{a}$ & Value \pm Error & Ref. $^{b}$ \\
\hline$P_{\mathrm{s}}$ & $(4.522 \pm 0.024) \mathrm{d}$ & L09 \\
Spectral type & G7V & B08 \\
\hline \hline Planet $^{c}$ & Value \pm Error & Ref. \\
\hline$P_{\mathrm{p}}$ & $(1.7429964 \pm 0.0000017) \mathrm{d}$ & A08 \\
$T_{\mathrm{c}}[\mathrm{BJD}]$ & $(2454237.53362 \pm 0.00014) \mathrm{d}$ & $\mathrm{A} 08$ \\
$i$ & $(87.84 \pm 0.10)^{\circ}$ & A08 \\
$R_{\mathrm{p}} / R_{\mathrm{S}}$ & $(0.1667 \pm 0.0006)$ & A08 \\
$a / R_{\mathrm{S}}$ & $(6.70 \pm 0.03)$ & A08 \\
$u_{\mathrm{a}}, u_{\mathrm{b}}$ & $(0.41 \pm 0.03),(0.06 \pm 0.03)$ & A08 \\
\hline
\end{tabular}

${ }^{a} P_{\mathrm{s}}-$ stellar rotation period; ${ }^{b}$ taken from Lanza et al. (2009) [L09], Alonso et al. (2008) [A08], or Bouchy et al. (2008) [B08]; ${ }^{c} P_{\mathrm{p}}-$ orbital period, $T_{\mathrm{c}}$ - central time of first transit, $i$ - orbital inclination, $R_{\mathrm{p}}, R_{\mathrm{S}}-$ planetary and stellar radii, $a$ - semi major axis of planetary orbit, $u_{\mathrm{a}}, u_{\mathrm{b}}$ - linear and quadratic limb darkening coefficients.

to be resolved by CoRoT. According to Alonso et al. (2008), this secondary contributes a constant $(5.6 \pm 0.3) \%$ of the total CoRoT-measured flux. CoRoT-2b's orbital period of $\approx 1.74$ days is about one third of CoRoT-2a's rotation period, and the almost continuous CoRoT data span 142 days, sampling about 30 stellar rotations and more than 80 transits. The light curve shows clear evidence of strong activity: there is substantial modulation of the shape on timescales of several days, and the transit profiles are considerably deformed as a consequence of surface inhomogeneities (Wolter et al. 2009).

Our data reduction starts with the results provided by the CoRoT N2 pipeline (N2_VER 1.2). CoRoT provides three-band photometry (nominally red, green, and blue), which we extend by a virtual fourth band resulting from the combination (addition) of the other bands. This "white" band is, henceforth, treated as an independent channel, and our analysis will mainly refer to this band. It provides the highest count rates and, more 
importantly, is less susceptible to instrumental effects such as long-term trends and "jumps" present in the individual color channels.

In all bands, we reject those data points flagged as "bad" by the standard CoRoT pipeline (mostly related to the South Atlantic anomaly). The last step leaves obvious outliers in the light curves. To remove them, we estimate the standard deviation of the data point distribution in short $(\approx 3000 \mathrm{~s})$ slices and reject the points more than $3 \sigma$ off a (local) linear model. Inevitably, we also remove a fraction of physical data (statistical outliers) in this step, but we estimate that loss to be less than a percent of the total number of data points, which we consider acceptable.

In all bands apart from the white, we find photometric discontinuities (jumps), which are caused by particle impact on the CoRoT detector. In the case of CoRoT-2a, the jumps are of minor amplitude compared to the overall count rate level, and we correct them by adjusting the part of the light curve following the jump to the preceding level.

Finally, we correct the CoRoT photometry for systematic, instrumental trends visible in all bands apart from white. To approximate the instrumental trend, we fit the (entire) light curve with a second order polynomial, $q$, and apply the equation

$c_{\mathrm{corr}, i}=c_{\mathrm{o}, i} \cdot \frac{\bar{c}}{q_{i}}$,

where $c_{\mathrm{o}, i}$ is the $i$ th observed data point, $q_{i}$ is the associated value of the best-fit second order polynomial, $\bar{c}$ represents the mean of all observed count rates in the band, and $c_{\mathrm{corr}, i}$ the corrected photometry.

The resulting light curve still shows a periodic signal clearly related to the orbital motion of the CoRoT satellite. This is again a minor effect in the white band, and we neglect this in the context of the following analysis.

In a last step, we subtract $5.6 \%$ of the median light curve level to account for the companion contribution. We use the same rule for all bands, which is only an approximation because, as Alonso et al. (2008) point out, the companion has a later type (probably $\mathrm{K}$ or $\mathrm{M}$ ) and, therefore, a different spectrum from CoRoT-2a.

\section{Analysis}

\subsection{Transit profiles and stellar activity}

A planet crossing the stellar disk imprints a characteristic transit feature on the light curve of the star (e.g., Pont et al. 2007; Wolter et al. 2009). The exact profile is determined by planetary parameters as well as the structure of the stellar surface. A model that describes the transit profile must account for both. One of the key parameters of the surface model is the limb darkening law. The presence of limb darkening seriously complicates transit modeling, because it can considerably affect the transit profile, while it is difficult to recover its characteristics from light curve analyses (e.g., Winn 2009).

Stellar activity adds yet another dimension of complexity to the problem, because a (potentially evolving) surface brightness distribution also affects the transit profiles. The local brightness on the surface can either be decreased by dark spots or increased by bright faculae compared to the undisturbed photosphere. Spots (or faculae) located within the eclipsed section of the stellar surface lead to a decrease (increase) in the transit depth, and the true profile depends on the distribution of those structures across the planetary path. Spots and faculae located on the non-eclipsed section of the surface do not directly affect the transit profile but change the overall level of the light curve. As transit light curves are, however, usually normalized with respect to the count rate level immediately before and after the transit, the non-eclipsed spot contribution enters (or can enter) the resulting curve as a time-dependent modulation of the normalized transit depth.

\subsection{Transit light-curve normalization}

As mentioned above, the normalization may affect the shape of the transit profiles. We now discuss two normalization approaches and compare their effect on the transit profiles. We define $f_{i}$ to be the measured flux in time bin $i, n_{i}$ an estimate of the count rate level without the transit (henceforth referred to as the "local continuum"), and $p$ a measure of the unspotted photospheric level in the light curve, i.e., the count rate obtained in the respective band, when the star shows a purely photospheric surface. Usually, the quantity

$y_{i}=f_{i} / n_{i}$

is referred to as the "normalized flux".

If we normalize the flux according to Eq. (2), we may produce variations in the transit light-curve depth in response to non-uniform surface flux distributions as encountered on active stars. To demonstrate this, we assume that a planet transits its host star twice. During the first transit, the stellar surface remains free of spots, but during the second transit there is a large active region on any part of the star not covered by the planetary disk (but visible). Consequently, the local continuum estimate, $n_{i}$, for the second transit is lower, and the normalized transit appears deeper, although it is exactly the same transit in absolute (nonnormalized) numbers.

To overcome this shortcoming, we define the alternative normalization to be

$z_{i}=\frac{f_{i}-n_{i}}{p}+1$

In both cases, the transit light curve is normalized with respect to the local continuum either by division or subtraction. The conceptual difference lies in the treatment of the local continuum level and how it enters the normalized transit light curve. Using Eq. (3), the observed transit is shifted, normalized by a constant, and shifted again. While the scaling in this case remains the same for all transits, the scaling applied in Eq. (2) is a function of the local continuum.

Following the above example, we assume that the same transit can be normalized by using Eqs. (2) and (3). To evaluate the differences between the approaches, we consider the expression

$\frac{z_{i}}{y_{i}}=\frac{\left(f_{i}-n_{i}\right) / p+1}{f_{i} / n_{i}} \geq 1$.

For $n_{i}=p$, Eq. (4) holds as a strict equality, i.e., both normalizations yield identical results. The inequality equates to true, if $p>n_{i}$ and $n_{i}>f_{i}$. The first condition reflects that the local continuum estimate should not exceed the photospheric light-curve level, and the second one says that the light-curve level is below the local continuum. The second condition is naturally fulfilled during a transit, and the first is also met as long as faculae do not dominate over the dark spots during the transit. In the case of CoRoT-2a, Lanza et al. (2009) find no evidence of a significant flux contribution due to faculae, so that we conclude that the normalized transit obtained using Eq. (3) is always shallower than that resulting from Eq. (2), unless $n_{i}=p$, in which case the outcomes are equal. 


\subsubsection{Quantifying the normalization induced difference in transit depth}

We now study a single transit and consider data points covered by index set $j$, for which the term $n_{j}-f_{j}$ reaches a maximal value of $T_{0}$ at some index value $j=T$. At this position, the normalization obtained from Eq. (3) is given by $z_{T}=\left(f_{T}-n_{T}\right) / p+1=-T_{0} / p+1$, whereas Eq. (2) yields $y_{T}=f_{T} / n_{T}=\left(n_{T}-T_{0}\right) / n_{T}$. These values are now used to compare the transit depths provided by the two normalizations. We note that we assume that the normalized depth is maximal at index $T$; this is always true for Eq. (3), but not necessarily for Eq. (2), a point that we assume to be a minor issue. We again find that $z_{T}=y_{T}$ if $n_{T}=p$. If, however, the local continuum estimate is given by $n_{T} \approx \alpha p(\alpha \leq 1)$, the results differ by

$z_{T}-y_{T}=T_{0} p^{-1}\left(\alpha^{-1}-1\right)$.

Using the extreme values observed for CoRoT- $2 \mathrm{a}(\alpha \approx 0.96$ and $\left.T_{0} \approx 0.03 \times p\right)$, the right-hand side of Eq. (5) yields $\approx 1.3 \times 10^{-3}$ for the difference in transit depth, caused exclusively by applying two different normalization prescriptions.

\subsubsection{Which normalization should be used?}

For planetary research it is important to "clean" the transit light curves of stellar activity before deriving the "undisturbed" profile associated with the planet only. Since transit light curves normalized using Eq. (3) are all scaled using the same factor, they preserve their shape and depth (at least relative to each other) and can, therefore, be combined consistently, which is not necessarily the case when Eq. (2) is used. This does not mean that the obtained transit depth is necessarily the "true" depth, because Eq. (3) includes the photospheric brightness level, $p$, as a timeindependent scaling factor. At least in the context of the lightcurve analysis, $p$ cannot be determined with certainty since the star may not show an undisturbed surface during the observation, which may actually never be shown.

A problem evident in CoRoT light-curve analyses is the existence of long-term instrumental gradients in the data (cf. Sect. 2). By modeling these trends with a "sliding" response, $R_{\mathrm{d}}$, of the detector, so that the relation between "true" photometry, $c_{i}$, and observation, $c_{\mathrm{o}, i}$, is given by $c_{i, \mathrm{o}}=c_{i} \cdot R_{\mathrm{d}, i}$, we find that Eq. (1) yields

$c_{\mathrm{corr}, i}=c_{i} \cdot\left(R_{\mathrm{d}, i} \frac{\bar{c}}{q_{i}}\right)$.

Obviously, the true photometry is recovered when the embraced term equates to one. However, the scaling of $\bar{c}$ in Eq. (1) is arbitrary, so that this is not necessarily the case. As long as $q_{i}$, however, appropriately represents the shape of $R_{\mathrm{d}, i}$, the term provides a global scaling, which cancels out in both of the Eqs. (2) and (3).

For our transit analysis, we argue in favor of the normalization along Eq. (3). We estimate the photospheric level from the highest count rate during the most prominent global maximum (at JD $\approx 2454373.3$ ) in each individual band. These estimates are based on the reduced light curves; in particular, we have accounted for both the instrumental trend and the stellar companion. Throughout our analysis, we use the values $p_{\text {white }}=$ $703000, p_{\text {red }}=489000, p_{\text {green }}=88500$, and $p_{\text {blue }}=124500$ (in units of $\left.\mathrm{e}^{-} / 32 \mathrm{~s}\right)$. Since even at that time, spots are likely to have been present on the stellar disk, these estimates might represent lower limits to the true value of $p$.

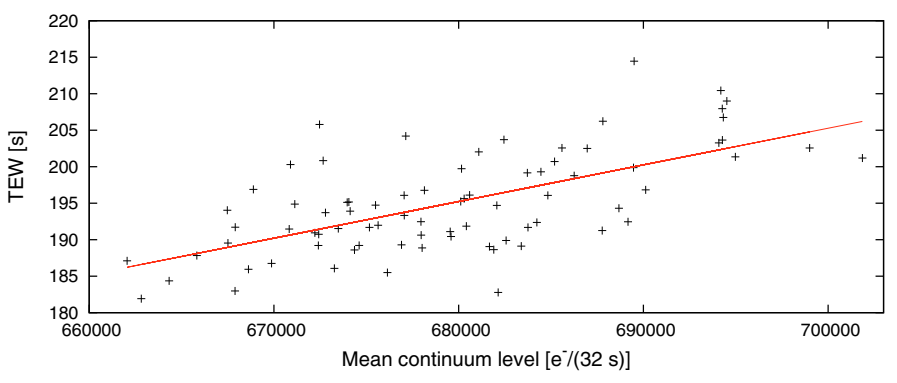

Fig. 1. Transit equivalent width (TEW) versus transit continuum level as well as the best-fit linear model.

\subsection{Transit profiles in CoRoT-2a}

The global light curve of CoRoT-2a shows pronounced maxima and minima and a temporally variable amplitude of the global modulation (Alonso et al. 2008). It is natural to expect the spot coverage on the eclipsed section of the stellar surface to be smallest where the global light curve is found at a high level, and transit events occurring during those phases should, thus, be least contaminated with the effects of stellar activity. The opposite should be true for transits during low light-curve levels.

To quantify the impact of activity on the transit profile, we define the transit equivalent width (TEW) of transit $n$

$T E W_{n}=\int_{t_{I}}^{t_{I V}}\left(1-z_{n}(t)\right) \mathrm{d} t \approx \sum_{i}\left(1-z_{n, i}\right) \delta t_{i}$,

where $t_{I}$ and $t_{I V}$ must be chosen so that they enclose the entire transit. Extending the integration boundaries beyond the true extent of the transit does not change the expectation value of Eq. (7), but only introduces an extra amount of error. The nominal unit of the TEW is time.

\subsubsection{The relation between transit equivalent width and global light-curve modulation}

As outlined above, we expect activity to have greater impact when the overall light-curve level is low. When this is true, it should be reflected by a relation between the transit equivalent width and the transit continuum level (the overall light-curve level at transit time).

In Fig. 1, we show the distribution of TEWs as a function of the local continuum level for all 79 transits observed with a 32 s sampling. There is a clear tendency for larger TEWs to be associated with higher continuum levels, thus, providing obvious evidence of activity-shaped transit light curves. In the same figure, we also show the best-fit linear model relation, which has a gradient of $\mathrm{d}(\mathrm{TEW}) / \mathrm{d}(\mathrm{CL})=(5 \pm 1.5) \times 10^{-4} \mathrm{~s} /\left(\mathrm{e}^{-} 32 \mathrm{~s}\right)$.

To corroborate the reality of the above stated correlation, we calculated the correlation coefficient, $R$. Its value of $R=0.642$ confirms the visual impression of a large scatter in the distribution of data points (cf., Fig. 1). We estimate the statistical error for a single data point to be $\approx 0.1 \%$, so that the scatter cannot be explained by measurement errors. To check whether the continuum level and the TEW are independent variables, we employ a t-test and find the null hypothesis (independent quantities) to be rejected with an error probability of $1.8 \times 10^{-10}$, so that the correlation between the TEWs and the continuum level must be regarded as highly significant.

As a cross-check of the interpretation of this finding, we also investigated the distribution of TEWs against time, which shows no such linear relation $(R=0.110)$. Therefore, we argue that the 


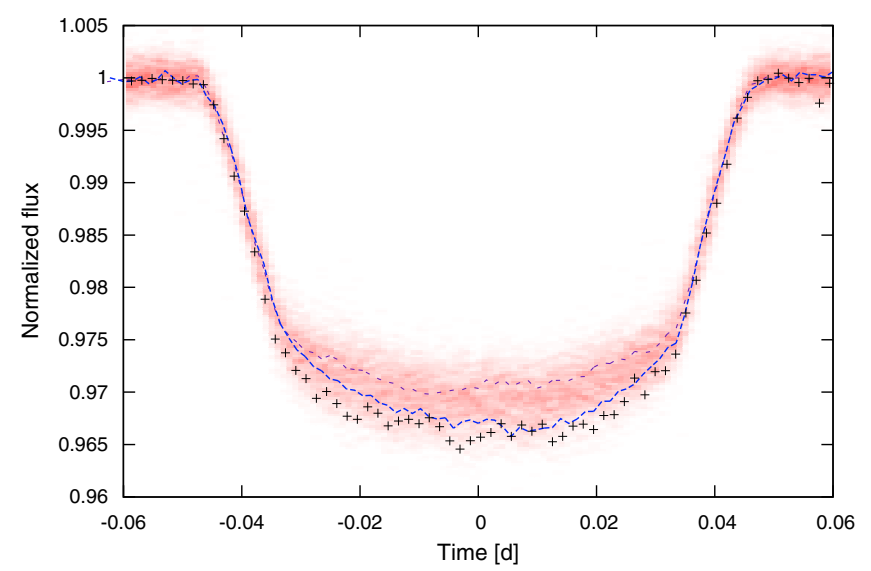

Fig. 2. Average transit light curves obtained by combining the ten profiles exhibiting the highest (thick dashes) and lowest (thin dashes) continuum levels. The crosses indicate our lower envelope estimate and the color gradient (red) illustrates the distribution of data points for all available transits.

effect is not instrumental or caused by our data reduction, but physical.

\subsection{Comparing high and low continuum level transits}

Since activity is evident in the profiles of the transit light curves, we further investigate its effect by comparing the most and least affected transit light curves. Therefore, we average the ten transits with the highest continuum levels (No. 3, 16, 42, 47, 50, 55, $68,73,76$, and 81) and compare the result to an average of the ten transits with the lowest continuum level (No. 15, 23, 35, 40, 43, 69, 72, 75, 77, and 80). In Fig. 2, we show the two averages as well as our computed lower envelope (see Sect. 3.5) superimposed on the entire set of folded photometry data points. The distribution of the entire set is denoted by a color gradient (red) with stronger color indicating a stronger concentration of data points. The curve obtained from the transits at a "low continuum state" is clearly shallower, as was already indicated by the TEW distribution presented in Fig. 1.

The difference in TEW amounts to $\approx 15.5 \mathrm{~s}$ in this extreme case. We checked the significance of this number with a Monte Carlo approach. On the basis of 20 randomly chosen transits, we constructed two averaged light curves using 10 transits for each and calculated the difference in TEW. Among 1000 trials, we did not find a single pair with a difference beyond $12 \mathrm{~s}$, so that the result is not likely to be caused by an accidental coincidence.

\subsection{Obtaining a lower envelope to the transit profiles}

As was demonstrated in the preceding section, activity shapes the transit light curves, and we cannot exclude that every transit is affected so that a priori no individual profile can be used as a template representing the "undisturbed" light curve. The distortion of the individual profiles is, however, not completely random, but the sign of the induced deviation is known as long as we assume that the dark structures dominate over bright faculae, which seems justified for CoRoT-2a (Lanza et al. 2009). In this case, activity always tends to raise the light-curve level and, thus, decreases the transit depth. Therefore, the most suitable model of the undisturbed profile can be estimated to be a lower envelope to the observed transit profiles.
We take a set of $N_{T}$ transit observations and fold the associated photometry at a single transit interval, providing us with the set $L C_{T, i}$ of transit data points. If the lower envelope were already among the set of observed transits, it would in principle look like every other light curve. In particular, it shows the same amount of intrinsic scattering (not including activity), characterized by the variance $\sigma_{0}^{2}$.

We estimate the variance to be

$\sigma_{0}^{2} \approx \frac{1}{N} \sum_{j}^{N}\left(L C_{T, j}-\mu_{j}\right)^{2}$

where $\mu_{j}$ is the (unknown) expectation value and $N$ is the number of data points. The aim of the following effort is to identify the lowest conceivable curve sharing the same variance. To achieve this, we divide the transit span into a number of subintervals, each containing a subsample, $s$, of $L C_{T}$. The distribution of data points in $s$ is now approximated by a "local model", $\operatorname{lm}(\gamma)$, with a free normalization $\gamma$; $l m$ can for instance be a constant or a gradient. Given $l m$, we adapt the normalization to solve the equation

$\left|\left(\frac{\sum_{\mathrm{s}}\left(L C_{\mathrm{s}}-\operatorname{lm}(\gamma)\right)^{2} \cdot H\left(\operatorname{lm}(\gamma)-L C_{\mathrm{s}}\right)}{\sum_{\mathrm{s}} H\left(\operatorname{lm}(\gamma)-L C_{\mathrm{s}}\right)}-\sigma_{0}^{2}\right)\right|=0$,

where $H$ denotes the Heaviside function $(H(x)=1$ for $x>0$, and $H(x)=0$ otherwise). In this way, we search for the local model compatible with the known variance of the lower envelope. The ratio on the left-hand side of Eq. (9) represents a variance estimator exclusively based on data points below the local model. It increases (strictly) monotonically except for the values of $\gamma$, where the local model "crosses" a data point and the denominator increases by one instantaneously. Therefore, there may be more than one solution to Eq. (9). From the mathematical point of view, all solutions are equivalent, but for a conservative estimate of the lower envelope the largest one should be used.

In Fig. 2, we show the lower envelope, which is in far closer agreement with the average of the high continuum transit profiles than with its low continuum counterpart. The derivation of the lower envelope is based on Eq. (9). To obtain an estimate of $\sigma_{0}^{2}$, we fitted a $500 \mathrm{~s}$ long span within the transit flanks $(3500 \pm 250 \mathrm{~s}$ from the transit center), where activity has little effect, with a straight line and calculated the variance with respect to this model. The resulting value (using normalized flux) of $\sigma_{0}^{2}=1.6 \times 10^{-6}$ was adopted in the calculation. Furthermore, we chose a bin width of $150 \mathrm{~s}$, and the "local model" was defined as a regression line within a $\pm 100 \mathrm{~s}$ time span around the bin center. Additionally, we postulated that at least 8 (out of $\approx 350$ ) data points per bin should be located below the envelope, which improved the stability of the method to the effect of outliers but has otherwise little impact.

\subsection{Transit profiles in different color channels}

CoRoT observes in three different bands termed "red", "green", and "blue". In the following, we present a qualitative analysis of the transit profiles in the separate bands. In the case of CoRoT-2a, approximately $70 \%$ of the flux is observed in the red band, and the remaining $30 \%$ is more or less equally distributed among the green and blue channels. To compare the profiles, we average all available transits in each band individually and normalize the results with respect to their TEW, i.e., after this step they all have the same TEW. The resulting profiles represent the curves that would be obtained if the stellar flux integrated along the planetary path was the same in all bands. 

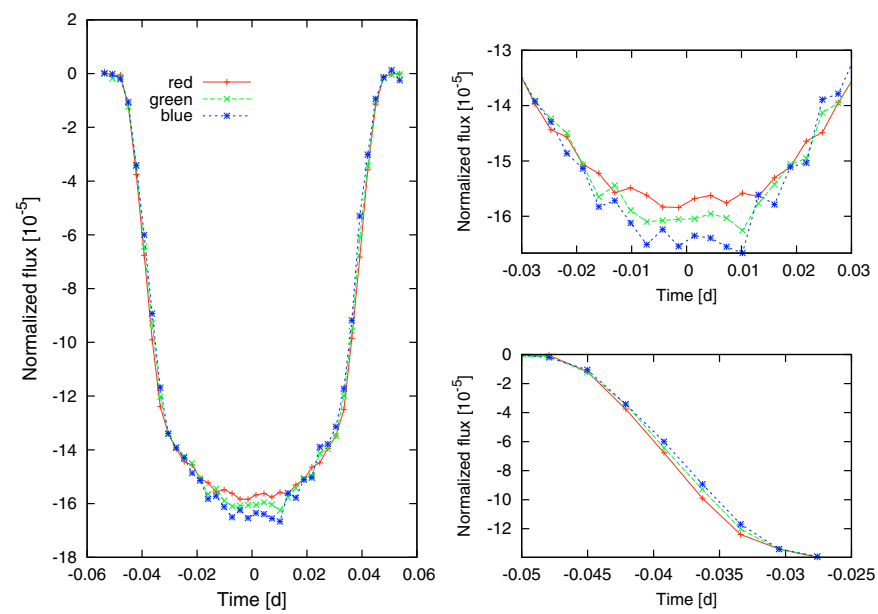

Fig. 3. Left panel: normalized transit in the three CoRoT bands red, green, and blue obtained by averaging all available data. Upper right: close-up of the transit center. Lower right: close-up of the ingress flank of the transit.

In Fig. 3, we show the transit light curves normalized in this way $(\mathrm{TEW}=1)$ obtained in the three bands.

The normalized transits show a difference in both their flank profile and their depth. The blue and green transit profiles are both narrower than the red one, and deeper at the center. This behavior is most pronounced in the blue band, so that the green transit light curve virtually always lies in-between the curves obtained in red and blue.

The behavior described above can be explained by a colordependent limb darkening law, with stronger limb darkening at shorter wavelengths as predicted by atmospheric models (Claret 2004) and observed on the Sun (Pierce \& Slaughter 1977). We checked that analytical transit models (Pál 2008) generated for a set of limb darkening coefficients, indeed, reproduce the observed behavior when normalized with respect to their TEW.

Normalizing the averaged transits not with respect to TEW but using Eq. (3) yields approximately the same depth in all bands, while the difference in the flanks becomes more pronounced. The reason for this could be an incorrect relative normalization, which can e.g., occur if the eclipsed section of the star is (on average) redder than the remainder of the surface because of pronounced activity or gravity darkening, or it may be a relic of an inappropriate treatment of the companion's flux contribution. Whatever the explanation, it is clear from Fig. 3 that the flanks and centers in the individual bands cannot be reconciled simultaneously by a renormalization. Therefore, our analysis shows that the transit light curves are color dependent.

\section{Stellar activity and planetary parameters}

The preceding discussion shows that stellar activity has a considerable influence on the profile of the transit light curves, and the derivation of the planetary parameters will therefore also be affected. We now determine the radius and the orbit inclination of CoRoT-2b taking activity into account, and discuss the remaining uncertainties in the modeling.

\subsection{Deriving the planetary radius and inclination from the lower envelope profile}

In the analysis presented by Alonso et al. (2008), the fit to the planetary parameters is based on the average of 78 transit light

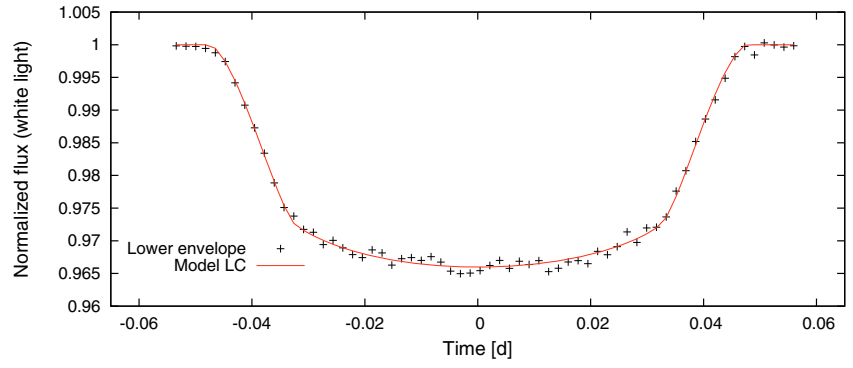

Fig. 4. Lower envelope of all normalized transit light curves (already shown in Fig. 2) and our model fit.

curves (see Table 1 for an excerpt of their results). While this yields a good approximation, the results still include a contribution of stellar activity, and an undisturbed transit is needed to calculate "clean" planetary parameters.

We follow a simplified approach to estimate the impact of activity on the planetary parameters. In particular, we use the lower envelope derived in Sect. 3.4 as the most suitable available model for the undisturbed transit. Starting from the results reported by Alonso et al. (2008), we reiterate the fit of the planetary parameters. In our approach, we fix the parameters of transit timing, i.e., the semi-major axis and stellar radius, and the limb darkening coefficients at the values given by Alonso et al. (2008) (cf. Table 1). The two free parameters are the planetary radius and its inclination.

We note that limb darkening coefficients recovered by light curve analyses are not reliable, especially when more than one coefficient is fitted (e.g., Winn 2009). However, since an accurate calibration of the CoRoT color bands is not yet available and the coefficients determined by Alonso et al. (2008) roughly correspond to numbers predicted by stellar atmosphere models ${ }^{1}$, we decided to use the Alonso et al. values, which also simplifies the comparison of the results.

For the fit, we use the analytical models given by Pál (2008) in combination with a Nelder-Mead simplex algorithm (e.g., Press et al. 1992).

The result of our modeling is illustrated in Fig. 4. The most probable radius ratio is $R_{\mathrm{p}} / R_{\mathrm{s}}=0.172 \pm 0.001$ at an inclination of $87.7^{\circ} \pm 0.2^{\circ}$. The quoted errors are statistical errors and only valid in the context of the model. These numbers should be compared with the values $R_{\mathrm{p}} / R_{\mathrm{S}}=0.1667 \pm 0.0006$ and $87.84^{\circ} \pm 0.1^{\circ}$ (cf., Table 1 ) derived without taking activity effects into account. The best-fit model inclination is compatible with the value determined by Alonso et al. (2008), but "our" planet is larger by $\approx 3 \%$. The planet's size depends mainly on the transit depth, which is, indeed, affected at about this level by both normalization (Sect. 3.2.1) and stellar activity (Sect. 3.4).

Clearly, the derived change in $R_{\mathrm{p}} / R_{\mathrm{S}}$ of 0.005 is much larger than the statistical error obtained from light-curve fitting, and, therefore, the neglect of activity leads to systematic errors in excess of statistical errors. While the overall effect in planet radius is $\approx 3 \%$, the error in density becomes $\approx 10 \%$. These errors are certainly tolerable for modeling planetary mass-radius relationships, but they are unacceptable for precision measurements of possible orbit changes in these systems.

\footnotetext{
${ }^{1}$ For $T_{\text {eff }}=5600 \mathrm{~K}$ and $\log (g)=4.5$, the PHOENIX models given by Claret (2004) yield quadratic limb darkening coefficients of $u_{\mathrm{a}}=0.46$ and $u_{\mathrm{b}}=0.25$ in the Sloan- $r^{\prime}$ band.
} 


\subsubsection{Planetary parameters and photospheric level}

As already indicated the normalization according to Eq. (3) relies on a "photospheric light-curve level", $p$, which enters as a global scaling factor and, therefore, also impedes the constraint of the planet's properties.

In a simple case, the star appears as a sphere with a purely photospheric surface, and the observed transit depth, $f_{0}$, can be identified with the square of the ratio of the planetary to the stellar radius

$f=\frac{\max \left(n_{i}-f_{i}\right)}{p}=\left(\frac{R_{\mathrm{p}}}{R_{*}}\right)^{2} L_{\mathrm{d}}$,

where $L_{\mathrm{d}}$ is a correction factor that accounts for limb darkening. However, when the observed star is active and the light curve is variable, there is no guarantee that the maximum point in the observed photometry is an appropriate representation of the photospheric stellar luminosity. Persistent inhomogeneities, such as polar spots and long-lived spot contributions, modulate the light curve, so that the pure photosphere might only be visible anytime the star is not observed or possibly never.

Assume our estimate, $p_{\mathrm{m}}$, of the photospheric level underestimates the true value, $p$, by a factor of $0<c \leq 1$ so that $p_{\mathrm{m}}=p \cdot c$ and $f_{l e, i}$ denotes the lower envelope transit light curve. The measured transit depth, $f_{\mathrm{m}}$, then becomes

$f_{\mathrm{m}}=\frac{\max \left(n_{i}-f_{l e, i}\right)}{p_{\mathrm{m}}}=\left(\frac{R_{\mathrm{p}}}{R_{*}}\right)^{2} \frac{L_{\mathrm{d}}}{c}$,

and another scaling factor must be applied to the radius ratio. While $p_{\mathrm{m}}$ is a measured quantity, $c$ is unknown, and if we neglect it in the physical interpretation, i.e., the right-hand side of Eq. (11), the ratio of planetary to stellar radius will be overestimated by a factor of $1 / \sqrt{c}$.

The value of $c$ cannot be quantified in the context of this work; only an estimate can be provided. Doppler imaging studies have found that polar spots are common and persistent structures in young, active stars (e.g., Huber et al. 2009). Assuming that polar spots also exist on Corot- $2 \mathrm{a}$ and that they reach to a latitude of $70^{\circ}$, they occupy roughly $2 \%$ of the visible stellar disk. Adopting a spot contrast of $50 \%, c$ becomes 0.99 in this case, and the planet size would be overestimated by $0.5 \%$. Since the poles of CoRoT-2a are seen under a large viewing angle, their impact would, thus, be appreciably smaller than the amplitude of the global brightness modulation (ca. 4\%). Nonetheless, in terms of sign, this effect counteracts the transit depth decrease caused by activity, and if the polar spots are larger or symmetric structures at lower latitudes contribute, it may even balance it.

\section{Discussion and conclusion}

Stellar activity is clearly seen in the CoRoT measured transit light curves of CoRoT-2a, and an appropriate normalization is necessary to derive the true transit light curve profile accurately.

The transit profiles observed in CoRoT-2a are affected by activity, as is obvious in many transits where active regions cause distinct "bumps" in the light curve (e.g., Wolter et al. 2009). Furthermore, our analysis indicates that not only profiles with bumps but presumably all transit profiles are influenced by stellar activity. This is evident in the relationship between the transit equivalent width and the level of the global light curve: transits observed during periods where the star appears relatively bright are deeper than those observed during faint phases. We demonstrated that this correlation is extremely significant, but also that the data points show a large scatter around an assumed linear model relation. If the star were to modulate its surface brightness globally and homogeneously, this relation would be perfectly linear except for measurement errors. Therefore, we interpret the observed scatter as a consequence of surface evolution. When the global light curve is minimal, we also find more spots on the eclipsed portion of the surface, but only on average, and for an individual transit, this may not be the case. Thus, the surface configuration is clearly not the same for every minimum observed.

In addition, we demonstrated that the transit profiles exhibit a color dependence compatible with a color-dependent limb darkening law as expected from stellar atmospheric models and the analogy with the solar case.

All these influences can potentially interfere with the determination of the planetary parameters. Using our lower (white light) transit envelope, we determined new values for the planetto-star radius ratio and the orbital inclination. While the latter remains compatible with previously reported results, the planet radius turns out to be larger (compared to the star) by about $3 \%$. Although our approach takes into account many activity-related effects, a number of uncertainties remain. For example, the photospheric light curve level needed for transit normalization cannot be determined with certainty from our analysis and the same applies to the limb darkening law. We are therefore more certain than for the planetary parameters themselves, in our conclusion that the errors in their determination are much larger than the statistical ones.

While CoRoT-2a is certainly an extreme example of an active star, stellar activity is a common phenomenon especially on young stars. Therefore, in general, stellar activity cannot be neglected in planetary research, if the accuracy of the results should exceed the percent level.

Acknowledgements. S.C. and U.W. acknowledge DLR support (50OR0105). K.H. is a member of the DFG Graduiertenkolleg 1351 Extrasolar Planets and their Host Stars. S.S. acknowledges DLR support (50OR0703).

\section{References}

Alonso, R., Auvergne, M., Baglin, A., et al. 2008, A\&A, 482, L21 Auvergne, M., Bodin, P., Boisnard, L., et al. 2009, ArXiv e-prints Bouchy, F., Queloz, D., Deleuil, M., et al. 2008, A\&A, 482, L25 Claret, A. 2004, A\&A, 428, 1001

Huber, K. F., Wolter, U., Czesla, S., et al. 2009, A\&A, accepted Lanza, A. F., Pagano, I., Leto, G., et al. 2009, A\&A, 493, 193 Pál, A. 2008, MNRAS, 390, 281

Pierce, A. K., \& Slaughter, C. D. 1977, Sol. Phys., 51, 25

Pont, F., Gilliland, R. L., Moutou, C., et al. 2007, A\&A, 476, 1347

Press, W. H., Teukolsky, S. A., Vetterling, W. T., et al. 1992, Numerical recipes in C. The art of scientific computing, ed. W. H. Press, S. A. Teukolsky, W. T. Vetterling, \& B. P. Flannery

Winn, J. N. 2009, in IAU Symposium, 253, IAU Symp., 99

Wolter, U., Schmitt, J. H. M. M., Huber, K. F., et al. 2009, A\&A, accepted 